\title{
The burst of solar and geomagnetic activity in August-September 2005
}

\author{
A. Papaioannou ${ }^{1}$, H. Mavromichalaki ${ }^{1}$, E. Eroshenko ${ }^{2}$, A. Belov ${ }^{2}$, and V. Oleneva ${ }^{2}$ \\ ${ }^{1}$ Nuclear and Particle Physics Section, Department of Physics, University of Athens, 15771 Athens, Greece \\ ${ }^{2}$ Institute of Terrestrial Magnetism Ionosphere and Radio wave Propagation (IZMIRAN) 142092, Troitsk, \\ Moscow Region, Russia
}

Received: 23 July 2008 - Revised: 26 November 2008 - Accepted: 12 December 2008 - Published: 2 March 2009

\begin{abstract}
During the August-September 2005 burst of solar activity, close to the current solar cycle minimum, a significant number of powerful X-ray flares were recorded, among which was the outstanding X17.0 flare of 7 September 2005. Within a relatively short period (from 22 August to 17 September) two severe magnetic storms were also recorded as well as several Forbush effects. These events are studied in this work, using hourly mean variations of cosmic ray density and anisotropy, derived from data of the neutron monitor network. During these Forbush effects the behavior of high energy cosmic ray characteristics (density and anisotropy) is analyzed together with interplanetary disturbances and their solar sources, and is compared to the variations observed in geomagnetic activity. A big and long lasting $(\sim 6 \mathrm{~h})$ cosmic ray pre-decrease $(\sim 2 \%)$ is defined before the shock arrival on 15 September 2005. The calculated cosmic ray gradients for September 2005 are also discussed.
\end{abstract}

Keywords. Interplanetary physics (Cosmic rays; Energetic particles) - Solar physics, astrophysics, and astronomy (Flares and mass ejections)

\section{Introduction}

The descending phase of solar cycle 23 evolved into a succession of bright manifestations of solar activity (e.g. Mavromichalaki et al., 2005; Belov et al., 2005; Papaioannou et al., 2009; Eroshenko et al., 2004). The solar activity increase in August-September 2005 turned out to be one of the last in the series of significant bursts during this current

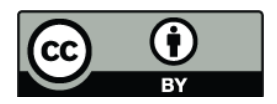

Correspondence to: $\mathrm{H}$. Mavromichalaki (emavromi@phys.uoa.gr) cycle. In less than one month (from 22 August to 17 September) $30 \mathrm{M}$-class and $11 \mathrm{X}$-class solar X-ray flares (SF) were recorded. Out of these 41 events, the largest one was the X17.0 flare, occurring on 7 September. This flare is the fifth most powerful flare recorded since regular X-ray observations began in 1975. The majority of flares in September 2005 occurred at the eastern part of the visible solar disk. The X17.0 SF originated at longitude $77^{\circ} \mathrm{E}$ and two other powerful SFs (X3.6 and X6.2) appeared at longitude $66^{\circ} \mathrm{E}$ on 9 September. These events are associated with the same active region on the Sun (AR10808). Normally the proton fluxes from such remote eastern flares are not observable near Earth (Belov, 2008; Eroshenko et al., 2004). However, in the case under consideration at least two of these eastern flares were followed by significant proton enhancements near Earth. The proton flux for $>10 \mathrm{MeV}$ particles increased up to $1000 \mathrm{pfu}$ on $10 \mathrm{September} \mathrm{(http://spidr.ngdc.noaa.gov;}$ http://www.sec.noaa.gov). Such great proton fluxes originating from eastern flares (even when considering all flares with $>45^{\circ} \mathrm{E}$ longitudes) have never before been observed near Earth. The proton flux registered by Rosetta/SREM, located $30^{\circ}$ east of Earth at a distance $\sim 1.3 \mathrm{AU}$, reached $\sim 100000 \mathrm{pfu}$ (Keil, 2005) at the beginning of 9 September at energies $>10 \mathrm{MeV}$. Proton flux at energies $>100 \mathrm{MeV}$ recorded on GOES, exceeded the value of $7 \mathrm{pfu}$ at this time. Thus, an unusually effective acceleration of solar particles may apparently be assumed in this case, probably including particles with energies $>1 \mathrm{GeV}$, even though such high energies did not reach Earth, because of the remote eastern source location.

In this paper, we present a complex analysis of these unusual effects, especially in cosmic rays (CR) of high energies (around $10 \mathrm{GeV}$ ). We reveal properties of $\mathrm{CR}$ variations associated with sporadic phenomena on the Sun during that

Published by Copernicus Publications on behalf of the European Geosciences Union. 
period using data obtained by the ground level neutron monitor network (http://cr0.izmiran.rssi.ru/common/links.htm). The main signature in the CRs, reflecting sporadic phenomena on the Sun and in interplanetary space, which we study here, is the Forbush effect (FE). The FE is the response of cosmic rays to the propagating disturbance including precursors (pre-increase and pre-decrease in CR variations before the main FE phase), CR intensity decrease as the main phase, and the recovery phase while the Earth exits from a disturbance area (Belov et al., 2007). Precursory decreases (predecrease) apparently results from a "loss-cone" effect, in which a neutron monitor station is magnetically connected to the cosmic ray-depleted region (Leerungnavarat et al., 2003, and references there). Pre-increase is usually caused by particles reflecting from the approaching shock. Sometimes in the analysis we use the term "Forbush decrease" (FD) which means the main phase of the FE when the CR density decrease is observed.

Section 2 presents the solar phenomena occurring during August and September 2005. In the following section the interplanetary disturbances and geomagnetic activity as a consequence of solar activity are presented. Section 4 briefly describes the results of the interplanetary perturbations in the CR behavior during August-September 2005. Section 5 describes the methods of analysis used in this paper. Results of analysis and discussion of the possible physical reasons are presented in Sect. 6. Then, in Sect. 7 a short conclusion is given.

\section{Solar activity}

\subsection{August 2005}

Two periods of strongly increased solar activity can be pointed out in this month: 1-3 August and 22-28 August. The first period was marked by three M-class solar flares. Specifically, the dominant active region AR 10794 produced a class M1.0 flare on 1 August at 13:00 UT $\left(13^{\circ} \mathrm{N} 32^{\circ} \mathrm{E}\right)$. The same active region was responsible for the M4.2 flare on 2 August and M3.4 flare on 3 August, both accompanied by coronal mass ejections (CMEs). Active region (AR) 10792 decayed continuously from 4 August until it disappeared behind the western limb of the Sun.

AR 10798 was identified on 15 August and evolved rapidly up to 20 August. It was responsible for the M2.6 and M5.6 flares on 22 August, and M2.7 flare on 23 August, peaking, respectively, at 01:32 UT, 17:27 UT and 14:44 UT. All three flare events had halo CMEs (http://lasco-www.nrl. navy.mil) associated with them which were later detected as interplanetary disturbances by the ACE spacecraft located at the Lagrange L1 point (the first two in quick succession of shocks recorded on 24 August and the third one on $25 \mathrm{Au}$ gust).

Ann. Geophys., 27, 1019-1026, 2009

\subsection{September 2005}

The dominating active region in September, AR 10798, also produced the main solar activity in the second part of $\mathrm{Au}$ gust. While this AR was at the non-visible side of the Sun it still produced some significant solar events from Earth's perspective. On 5 September, a long duration C-class flare was recorded by GOES and a CME was associated with it. The next day (6 September), a long duration M1.4 flare peaking at 22:02 UT was recorded. The source region was still located beyond the east limb. Finally, on 7 September, the sunspot group became visible again (AR 10798 which after its rotation was named AR 10808). This sunspot group proved to be extremely active: a class X17 flare was recorded by GOES on 7 September, peaking at 17:40 UT. A type II radio burst was also detected, indicating the presence of a CME. Unfortunately, data from LASCO and EIT were absent during that time and as a consequence no estimates could be made of the speed and strength of the associated CME. Due to the position of the sunspot group at that moment, it can be assumed that the associated CME was mainly directed eastwards. This group continued its activity on 8 September with two flares (M2.1 and X5.4). On 9 September, five M-class flares followed, as well as the X1.1 and X3.6 flare and at the end of the day a X6.2 flare which peaked at 20:04 UT. This latter flare was especially important since a strong CME was associated with it. On 10 September, three M-class flares occurred as well as an X1.1 and X2.1 flare (these latter two associated with a CME). The next day (11 September), the group was responsible for two M-class flares (M3.4 and M3.0). The last flare peaking at 13:12 UT had an associated CME. On 12 September, four M-class flares were measured by GOES. Three peaks were observed in the GOES X-ray time profiles indicating X1.5, X1.4 and X1.7 flare maxima at 19:27 UT, 20:04 UT and 23:22 UT, respectively. A halo CME was associated with this event since the sunspot group at that moment was located at central meridian. The group stayed active and produced five more M-class flares (one was the M9.8 flare on 17 September) and the X1.1 flare on 15 September. The list of the most significant solar flares during August-September with their characteristics is given in Table 1 .

\section{Interplanetary disturbances and geomagnetic activity}

\subsection{August 2005}

Four periods of strong geomagnetic activity can be identified in August 2005: 6-7 August, 10 August, 24-25 August and 31 August. Particularly, the arrival of a fast solar wind stream on 5 August induced active to minor storm conditions with a peak $K_{p}$ index of 5 - on 6 August, because of a negative $B_{z}$ component of the interplanetary magnetic field (IMF). Early on 10 August, a very short (several hours) and small increase of the IMF (up to $9 \mathrm{nT}$ ) with a negative $B_{z}$ component led to a 
Table 1. The most significant solar flare events related to cosmic ray irregular behavior during August-September 2005. Solar flare date, onset time and class (importance), as well as heliographic location (latitude and longitude) on the face of the Sun are listed for each event.

\begin{tabular}{ccccc}
\hline \multirow{2}{*}{ Date } & Onset time (UT) & \multicolumn{2}{c}{ Associated solar flares } \\
Importance & Latitude $\left(^{\circ}\right)$ & Longitude $\left(^{\circ}\right)$ \\
\hline 22 Aug 2005 & $16: 46$ & M5.6 & $13^{\circ} \mathrm{S}$ & $65^{\circ} \mathrm{W}$ \\
7 Sep 2005 & $17: 17$ & $\mathrm{X} 17$ & $11^{\circ} \mathrm{S}$ & $77^{\circ} \mathrm{E}$ \\
9 Sep 2005 & $19: 13$ & $\mathrm{X} 6.2$ & $12^{\circ} \mathrm{S}$ & $67^{\circ} \mathrm{E}$ \\
10 Sep 2005 & $21: 30$ & $\mathrm{X} 2.1$ & $13^{\circ} \mathrm{S}$ & $47^{\circ} \mathrm{E}$ \\
13 Sep 2005 & $19: 19$ & $\mathrm{X} 1.5$ & $09^{\circ} \mathrm{S}$ & $10^{\circ} \mathrm{E}$ \\
\hline
\end{tabular}

slightly disturbed geomagnetic situation $\left(K_{p}\right.$-index reached only the value of 4).

The most intensive magnetic storm was recorded on 24 25 August with a peak $K_{p}$-index of -9 and a $D_{s t}$ index of $-216 \mathrm{nT}$. Three CMEs were the cause of this extreme activity. The first CME left a signature in the ACE data just before 06:00 UT, the second around 09:00 UT. Following the arrival of the second CME the IMF $B_{z}$ value reached $\sim-40 \mathrm{nT}$ while the solar wind speed increased to over $700 \mathrm{~km} / \mathrm{s}$. Shortly afterwards, the $K_{p}$ value increased to 7 and then to 9 , and it remained at minor to severe storm levels until early 25 August. A third CME was detected as a slight strengthening of IMF at 13:00 UT (6.4 nT) on $25 \mathrm{Au}$ gust and the $K_{p}$ index reached the level of minor magnetic storm at 15:00-18:00 UT. At the end of 30 August a sudden storm commencement (SSC) was registered (IMF was $18.6 \mathrm{nT}$, solar wind velocity $V_{s w}$ increased up to $512 \mathrm{~km} / \mathrm{s}$ ) although the storm developed towards the middle of $31 \mathrm{Au}$ gust, and reached a level of a strong magnetic storm $\left(K_{p}=7\right)$. It was followed by a small FE $(\sim 1 \%)$, thus, we do not consider this case in this study.

\subsection{September 2005}

Geomagnetic conditions in September 2005 were determined by the arrival of five halo CMEs recorded on 7, 9, 10, 11 and 13 September. These CMEs were associated with the large solar flares of this period. Clear shocks were seen in different physical quantities (e.g. solar wind speed $V_{s w}$, IMF intensity $B$ and $B_{z}$ ) measured by the ACE spacecraft. Specifically, on 9 September, around 13:15 UT, the $V_{s w}$ jumped from 350 to $500 \mathrm{~km} / \mathrm{s}$, the peak IMF was of $20 \mathrm{nT}$ and $B_{z}$ went down to $-10 \mathrm{nT}$. Two days later, on 11 September, a shock arrived just before 01:00 UT. The IMF magnitude increased to $18.2 \mathrm{nT}$ with a significant southward component $\left(B_{z}=-6.4 \mathrm{nT}\right)$ and the maximum solar wind speed was $1059 \mathrm{~km} / \mathrm{s}$. Later, on 12 September, after 06:00 UT, the total IMF increased from 6 to $10 \mathrm{nT}$, the $B_{z}$ value went down to $-10 \mathrm{nT}$ and the $V_{s w}$ increased from 700 to $993 \mathrm{~km} / \mathrm{s}$. Finally, on 15 September after two SSCs were recorded at 08:35 and 09:04 UT, the solar wind speed rose from 550 to $862 \mathrm{~km} / \mathrm{s}$, $B_{z}$ became $-7 \mathrm{nT}$ and the total IMF went up to $17.8 \mathrm{nT}$.
Related to the arrival times of the CME shock fronts, the geomagnetic consequences were as follows: as a result of the CME on 7 September, associated with the remote eastern X17 flare, the $K_{p}$ index increased to 4 on 9 September and to 5 on 10 September. On 11 September, the $K_{p}$ peaked to 9 as the geomagnetic field was further disturbed by the arrival of the CME of 9 September associated with the X6.2 flare. In the declining phase of the passage of these CMEs (on 9, 10,11 September), the geomagnetic storm received a boost at the arrival of a shock front on 12 September. As a result, the planetary $K_{p}$ index reached a value of -6 . On 15 September the interplanetary disturbance induced by the arrival of the full halo CME on 13 September, caused the major geomagnetic storm $\left(K_{p}=7\right)$ which faded away on 16 September as $K_{p}$ became equal to 4 .

\section{Cosmic ray variations}

Significant galactic cosmic ray (CR) variations were observed as a consequence of the August and September 2005 solar activity. In order to study these variations, hourly values of CR density, vector anisotropy and CR gradients derived from data of the neutron monitor network (http://cr0.izmiran. rssi.ru/common/links.htm) have been used. Among numerous effects in the CRs during those two months, three Forbush effects are noticeable: 24-25 August (amplitude 6.4\% for CR with rigidity $10 \mathrm{GV}), 11$ September $(12.1 \%)$ and 15 September $(5.1 \%)$. Contradictory to the situation of July 2005, when powerful flares occurred on the western limb (Papaioannou et al., 2005, 2009), the events of this period turned out to be much more geoeffective. As noted in Sect. 3, very fast shocks arrived at the Earth and their mean velocity exceeded $1100 \mathrm{~km} / \mathrm{s}$ in all three cases (see Table 2). In addition, on 15 September a big and long lasting pre-decrease in CR density was observed prior to the shock arrival. The list of basic events which have been used for our analysis is presented in Table 2. 


\section{Data analysis}

\subsection{Data used}

In this study, data from the IZMIRAN database on the Forbush effects and interplanetary disturbances is used. This data base is not yet available on the Internet. Sources of this data base include calculations of the $\mathrm{CR}$ variation parameters (hourly values), GOES measurements (continuously updated), and the OMNI data base from the following web sites: http://cr20.izmiran.rssi.ru/AnisotropyCR/ Index.php, http://www.ngdc.noaa.gov/, http://omniweb.gsfc. nasa.gov/ow.html, http://sec.ts.astro.it/sec_ui.php, and CME data from http://lasco-www.nrl.navy.mil. The list of SSCs has also been used (ftp://ftp.ngdc.noaa.gov/STP/SOLAR DATA/SUDDEN_COMMENTS/) as a proxy for interplanetary shocks - the time of the SSC generally defines the onset of a FE.

Data from the entire global network of neutron monitors (40-45 stations of ground level CR observations, distributed on the globe) (http://cr0.izmiran.rssi.ru/common/links.htm) make up another IZMIRAN database which is used for the CR parameter calculations. Except for the CR current measurements, this database includes also the unique properties of each station: coupling coefficients, asymptotic directions and yield functions.

Parameters used for our analysis include CR density (A0) and anisotropy (Axy equatorial component of the first harmonic of anisotropy), solar wind data (velocity and density), interplanetary magnetic field intensity $\left(B_{\text {imf }}\right.$ and $\left.B_{z}\right)$, as well as solar data and geomagnetic activity indices $\left(K_{p}\right.$ and $\left.D_{s t}\right)$.

\subsection{Analysis methods}

In order to obtain the flux variations (A0) and the first harmonic of anisotropy (Axy) for $10 \mathrm{GV}$ cosmic rays, above the magnetosphere, data from as many stations as possible from the entire global network of neutron monitors has been used. The calculation of CR density and anisotropy components has been performed using the Global Survey Method (GSM) (e.g. Belov et al., 2005, 2007; Asipenka et al., 2009). We simulate the expected CR intensity at every station, taking into account the property of this station (cut off rigidity, altitude, asymptotic directions) by means of special coupling functions and coefficients (e.g. Belov et al., 2005b). As a result of the best fitting to real data, we obtain a set of $\mathrm{CR}$ characteristics (density and components of the first harmonic of anisotropy) at definite rigidities $(R)$ beyond the magnetosphere. In our database, we use GSM results for $10 \mathrm{GV}$ (http://cr20.izmiran.rssi.ru/AnisotropyCR/Index.php) since this rigidity is close to the effective rigidity of the majority of high and mid-latitude neutron monitors (NM) (effective rigidity is that upon which the variation of primary CRs is equal to variations recorded by the separate NM). The CR density variations, obtained for $10 \mathrm{GV}$ rigidity have high ac- curacy and reflect all solar wind disturbances responsible for the FEs.

Hourly means of CR density and components of the first spherical harmonic of CR anisotropy, derived in such a way, are global CR characteristics, beyond the magnetosphere, and do not depend on the local position of detectors - this is the first advantage of this method compared to other approaches where the researchers simply use the analyses count rate from individual stations. The second advantage is that these characteristics are obtained with high accuracy ( $\sim 0.05 \%$ for hourly means) that provides more precise estimations of the FE parameters.

Longitudinal and pitch-angle distributions of the CR variations have been calculated by the "ring" station method (Belov et al., 2001a, 2003). This is the method where the anisotropy is obtained by the data from high latitude neutron monitors with approximately similar features but with different longitudes. This approach allows the "picturing" of a longitudinal distribution of CR intensity at any moment in time. The CR density gradients have been calculated by the convection diffusion model of anisotropy as was introduced by Krymsky et al. (1964) and developed by Belov (1987) and by Chen and Bieber (1993). The implementation of this method is described in detail in Belov et al. (1987); Papaioannou et al. (2009).

For some estimates, we often use the parameter " $V B$ " which characterizes the modulating ability of the interplanetary disturbance (Belov et al., 1991b). It is a product of $V_{s w}$ maximum and $B_{\text {imf }}$ maximum within a disturbance, normalized to the quiescent conditions: $V B=\left(V_{\max } / 400 \mathrm{~km} / \mathrm{s}\right) \times\left(B_{\max } / 5 \mathrm{nT}\right)$. Normally, strong interplanetary disturbance $\left(B_{\max }\right)$, means greater parameter $V B$. And according to Belov et al. (1991b), FE amplitude $\left(A_{F E}\right)$ presents linear dependence on $V B$.

\section{Results and discussion}

Time profiles of solar wind (SW) speed, IMF intensity (IMF) and CR density (A0) as well as data of the equatorial component of anisotropy (Axy) and geomagnetic activity $\left(K_{p}\right.$ and $D_{s t}$ indices) for the period from 20 August to 29 September 2005, are plotted in Fig. 1. As can be seen by the evolution of all parameters, each episode of solar activity resulted in well pronounced disturbances in interplanetary space (jumps in the SW speed and IMF). Many shocks were produced and, despite their remote source location on the Sun, reached the Earth and caused strong geomagnetic storms (SSCs, behavior of $D_{s t}$ and $K_{p}$ indices). Each disturbance modulated the galactic cosmic rays (GCR) creating a series of significant FEs starting from 24 August. The main parameters, characterizing the situation on the Sun, in the interplanetary space, in the geomagnetic field and in the GCR during this period, are given in Tables 1 and 2. 
Table 2. Significant interplanetary disturbances, related Forbush effects and geomagnetic activity indices recorded during August-September 2005. Parameters include: Date: date of SSC; Time: SSC arrival time at Earth; $V_{m}$ : mean velocity of the disturbance propagation from Sun to the Earth; $V B$ : product maximum SW velocity Vmax and IMF intensity $B_{\max }$ in the considered disturbance; A0: maximum value of $\mathrm{CR}$ density variation during the FE (the magnitude of FD) beyond the magnetosphere, Axy: maximum equatorial component of the CR anisotropy during the FE by GSM method derived; $K_{p} \& D_{s t}$ : maximum values of geomagnetic activity indices.

\begin{tabular}{cccccccc}
\hline \multicolumn{3}{c}{ Interplanetary disturbances } & \multicolumn{3}{c}{ Forbush Effect (FE) } & $K_{p}$ & $D_{s t}$ \\
Date & Time (UT) & $V_{m}(\mathrm{~km} / \mathrm{s})$ & $V B$ & $\mathrm{~A} 0(\%)$ & Axy (\%) & & nT \\
\hline 24 Aug 2005 & $04: 43$ & 1113 & 18.7 & 6.4 & 2.61 & 8.67 & -216 \\
9 Sep 2005 & $14: 01$ & 931 & 3.21 & 3.2 & 2.37 & 5.67 & -60 \\
11 Sep 2005 & $01: 41$ & 1328 & 8.89 & 12.1 & 5.87 & 7.67 & -123 \\
12 Sep 2005 & $06: 00$ & 1282 & 4.77 & 5.1 & 2.87 & 7.00 & -84 \\
15 Sep 2005 & $09: 04$ & 1118 & 1.53 & 4.2 & 1.52 & 7.00 & -38 \\
\hline
\end{tabular}

A new rise in solar activity started with the M5.6 western flare $\left(65^{\circ} \mathrm{W}\right)$ on 22 August, leading to a series of disturbances in the interplanetary and near Earth space. In particular, the IMF intensity exceeded $40 \mathrm{nT}$ and the mean velocity of a CME propagation $\left(V_{m}\right)$ was $1113 \mathrm{~km} / \mathrm{s}$ on 24 August. This disturbance triggered an extreme geomagnetic storm with $K_{p}$-index around 9- and $D_{s t}$ index down to $-216 \mathrm{nT}$. The $V B$ product which characterizes the possible magnitude of the FE originating from such conditions (Belov et al., 2001b), had the very large value of 18.71 that means a strong distinction of real disturbed conditions from the normal. The magnitude of the FD on 24 August had the value of $6.4 \%$. Usually under such $V B$ conditions, the amplitude is expected to be larger. Nevertheless, it should be pointed out that this magnitude is one of the biggest recorded by all FEs associated with western near the limb flares over the last 40 years. Perhaps, before the onset of this FE, there was already a small effect in CR on 23 August caused by a disturbance from a halo CME associated with a remote western flare occurring at 01:32 UT on 22 August (M2.6). Two other shocks arrived towards the Earth and created two moderate magnetic storms $\left(K_{p} \sim 5-6\right)$ and two small Forbush decreases $\sim 2-2.5 \%$ on 31 August and 2 September, respectively. The picture of this disordered period was completed by a series of four FEs during 9-15 September. The first effect in this series is affiliated to the shock arrival on 9 September, which is associated with the X17.0 flare occurring on 7 September, at longitude $77^{\circ} \mathrm{E}$. Resembling the event of 24 August, this effect may also be considered as some deflection from the normal evolution of the FE. Earth again turned out to be at the periphery of the disturbance (at the western part this time) and the FE did not reach its peak value. If the flare had occurred closer to the center of the solar disk, one would have observed a giant Forbush decrease, but in the current case the rather far location of the eastern source did not allow one to observe the disturbance near Earth in full measure. The jump of the SW velocity and IMF intensity was very small, the amplitude of the FE was only $3.2 \%$ and the geomagnetic activity was moderate.

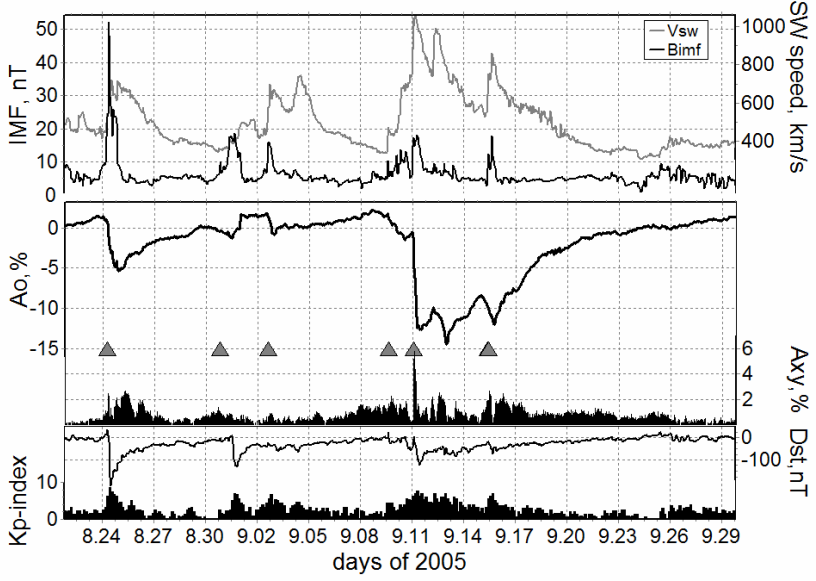

Fig. 1. Parameters of the solar wind (upper panel), density (A0) and anisotropy (Axy) of the $10 \mathrm{GV} \mathrm{CR}$ (mid panel), and $D_{s t}$ and $K_{p}$ indices of geomagnetic activity as well (lower panel) in AugustSeptember 2005. "SSC" corresponds to the moment of a shock arrival at the Earth.

In contrary to this effect, the next disturbance on September 11 , associated with the X6.2 flare at longitude $66^{\circ} \mathrm{E}$, created a strong geomagnetic storm and caused a large FE characterized by a fast decrease of CR intensity by $\sim 12 \%$ and a high anisotropy of GCR up to $5.8 \%$ in the equatorial component for particles of $10 \mathrm{GV}$. The mean velocity of shock propagation was $1328 \mathrm{~km} / \mathrm{s}$, the SW speed increased up to $980 \mathrm{~km} / \mathrm{s}$ and the IMF intensity peaked at $20 \mathrm{nT}$. The $V B$ parameter had the value 8.9 that corresponds to a magnitude of $\sim 12 \%$ of the Forbush decrease that was actually observed. Two more FEs of less magnitude (5.1 and 4.2\%) are noted in Fig. 1. These occurred in the minimum of the main effect from 11 September, and delayed its recovery.

As listed in Table 2, on 12 September, another disturbance arrived towards the Earth which was associated with a far eastern (47E) flare of X2.1 importance occurring at 21:30 UT on 10 September and followed by a CME with initial speed 


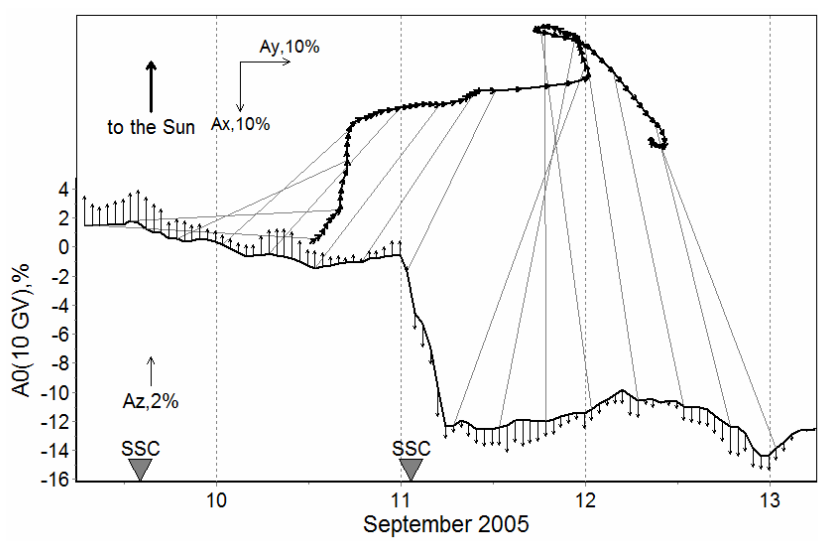

Fig. 2. Behavior of the CR density (0) and vector of equatorial component of the anisotropy (Axy) for $10 \mathrm{GV}$ rigidity CR during 9-13 September 2005. Vertical vectors represent the north-south component of the CR anisotropy. Triangles indicate the timing of the shock arriving. The thin lines connect the equal time moments on the density curve with the vector diagram.

of $1893 \mathrm{~km} / \mathrm{s}$. This CME propagated with a high mean speed ( $V_{m}=1283 \mathrm{~km} / \mathrm{s}$ ) from the Sun to the Earth contributing to the background of already disturbed conditions. By this time the solar wind velocity had increased to $997 \mathrm{~km} / \mathrm{s}$, a large magnetic storm was evolving at the Earth and the parameter $V B$ had the value of 4.77 which corresponds to $\sim 5 \%$ magnitude of the FE.

The series of FEs with corresponding onsets on 9, 11 and 12 September are presented in Fig. 2 where the CR density variation for every $6 \mathrm{~h}$ is connected to the corresponding time on the vector diagram of the equatorial component of anisotropy for these events. The behavior of the anisotropy vector clearly demonstrates a certain response to the arrival or approach of different disturbed structures. The first noticeable turn of the anisotropy vector Axy on 10 September coincides with the Earth entering the pronounced part of the interplanetary disturbance on 9 September $\left(B_{\text {imf }}\right.$ increased up to $15 \mathrm{nT}, V_{s w}$ reached the value $\sim 550 \mathrm{~km} / \mathrm{s}$ ). The next sharp turning of Axy occurred on 11 September in the end of decreasing phase of FE associated with a disturbance from 9 September (X6.2). And more sharp changes in the Axy vector behavior are related to the arrival of a new disturbance on 12 September.

The last FE in this series is related to a disturbance arriving on 15 September, after X1.5 flare occurred on 13 September. The associated shock did not produce strong changes in the interplanetary space parameters or in the geomagnetic activity, but led to a significant Forbush decrease (4.2\%). It is interesting to note that the shock arrived later than the onset in CR density decrease. Thus, this case appears to be a good example of a precursor to Forbush decrease (Belov et al., 2001a, 2003). In Fig. 3 the change of CR anisotropy direction starts from about 03:00 UT on 15 September - long

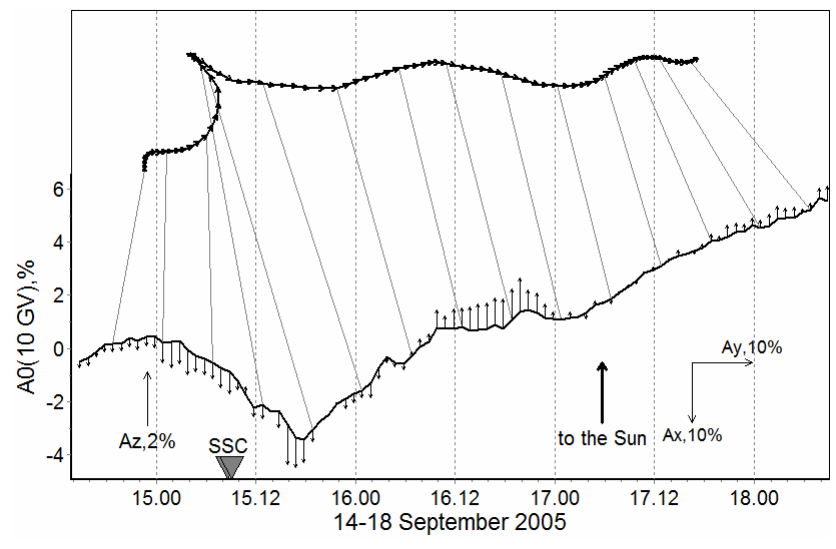

Fig. 3. Forbush effect on 15 September 2005: Behavior of the CR density and vector of equatorial component of CR anisotropy in the period from 15-18 September 2005. The dates are presented in the format dd.hh (days.hours). Designations are the same as in Fig. 2.

before the shock arrival (at 09:04 UT). After the minimum of FD, with the beginning of a recovery phase, the anisotropy vector takes an usual westward direction that is provided by a recovering flux from the eastern side. This direction remains for several days until the end of recovery of the CR intensity. In Fig. 4, the longitudinal distribution of CR variations in asymptotic longitudes obtained by the "ring station" method (Belov et al., 2001a) is plotted for 14-15 September. It is worth mentioning that around 03:00 UT on 15 September the narrow region of longitudes (in a sector $90^{\circ}-180^{\circ}$ ) with low CR intensity stands out against the background of increases in CR variations. This peculiarity became especially well pronounced from $\sim 06: 00 \mathrm{UT}$ ( $3 \mathrm{~h}$ prior to the SSC). As described in Belov et al. (1995, 2001a); Ruffolo et al. (1999), the pitch-angle distribution of the CR variations, before the disturbance arrival assumes a specific form when abrupt changes (from negative to positive or vice versa) of the $\mathrm{CR}$ variations occur at very close longitudes, near the magnetic force line of the IMF.

The CR gradients calculated by means of the convectiondiffusion model for this period presented interesting features. As can be seen in Fig. 5, an increase of the IMF strength is always followed by an increase of the CR gradients. This can be verified in the 24 August 2005 event which was a standard, well pronounced FE. In the complex situation of September 2005, when a series of FEs took place, the CR gradient behavior is rather different from the expected one. For example, on 10-13 September, while the IMF varies no more than to $15 \mathrm{nT}$ the gradients increase up to more than $300 \% / \mathrm{AU}$. The main reason for this is apparently not in the local (near Earth) conditions but in the conditions rather far from Earth where the strongest interplanetary disturbance and the minimum of CR density are located. It is this far CR decrease which provides a large $\mathrm{CR}$ gradient observable near Earth. Often the peripheral events behave in a similar way - the observed near 


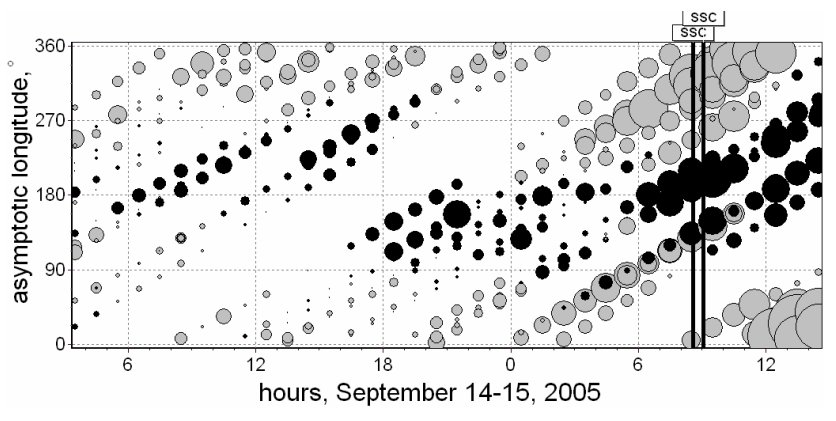

Fig. 4. Forbush effect on 15 September 2005: distribution of the CR variations by asymptotic longitudes after reduction of the isotropic part. The black circles signify a decrease of intensity and the gray circles an increase relatively to the base value before the FE. The size of the circle is proportional to the amplitude of CR variation. Vertical lines indicate the timing of the shocks (08:35 UT and 09:04 UT).

Earth CR gradient is much more than expected for such IMF intensity. In this aspect the event on 11 September looks like those of July 2005 (Papaioannou et al., 2009) with the only difference that in the considered case, the solar source of the disturbance is remote to the east.

\section{Conclusions}

Close to the solar cycle minimum, during August-September 2005, the observed solar activity burst caused a series of large modulation effects in CRs. Five CMEs (three eastern, one central and one western), all associated with powerful Xray flares, were the main phenomenon that caused the strong and large size perturbations observed in the inner heliosphere during this period of time.

Western (22 August) and eastern (7 September and 9 September) CMEs originated from very remote locations on the Sun, but significant effects in CR and in Earth's magnetosphere were recorded even in these cases.

A series of Forbush effects occurred, three of which were distinguished by the magnitude of CR density decrease: 2425 August (magnitude $6.4 \%$ for the CR with rigidity $10 \mathrm{GV}$ ), 11 September (12.1\%) and 15 September (5.1\%). In all three cases very fast shocks arrived at the Earth with a mean velocity of propagation exceeding $1100 \mathrm{~km} / \mathrm{s}$.

On 15 September, a big and long lasting pre-decrease in $\mathrm{CR}$ density preceded the shock arrival, which is also clearly shown in the longitude and pitch-angle distribution of the CR intensity.

Modulation effects in CRs, as FEs were large despite the fact that Earth often entered only a periphery of the propagating disturbance. This is an apparent evidence of much greater CR modulation taking part in the central part of the considered interplanetary disturbance, which is even confirmed by

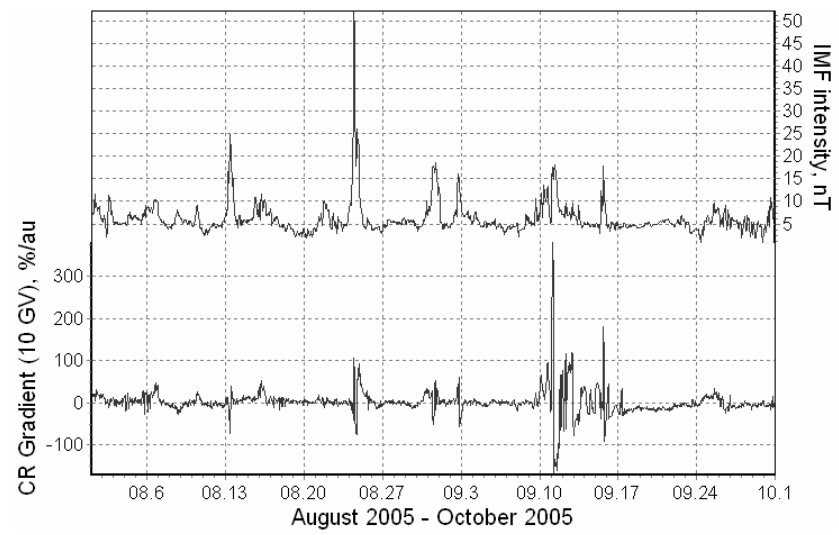

Fig. 5. The cosmic ray gradient calculated at $10 \mathrm{GV}$ (lower curve) and the IMF intensity (upper curve) are presented.

the calculated CR gradients despite the remoteness of the source.

In these events the CR effects observable near Earth give additional information about the size and the power of CMEs that may be useful for diagnostics of solar and interplanetary disturbances and for estimating their possible effect in the Earth's environment. Besides, the characteristic changes in a behavior of CR vector anisotropy may be used for an advance warning before the arrival of the interplanetary shock.

Acknowledgements. We are thankful to all collaborations providing continued ground-level monitoring of CRs and to all researchers presenting operational satellite data on the Internet. This work was supported by the RFBR grants 04-02-16763 and 05-0217251 , by the Program of RAN Presidium "Neutrino physics", by the "Real-Time Neutron Monitor Database-NMDB" project (FP7 grant 213007) funded by European Social Funds and National resources, and by the NSF grant ATM-0527878 (USA and Canadian NM stations). Thanks are due to the editor and to the anonymous referees for their useful comments.

Topical Editor R. Forsyth thanks N. B. Crosby and another anonymous referee for their help in evaluating this paper.

\section{References}

Asipenka, A. S., Belov, A. V., Eroshenko, E. A., Klepach, E. G., Oleneva, V. A., and Yanke, V. G.: Interactive database of cosmic ray anisotropy (DB A10), Adv. Space Res., 43, 708-716, 2009.

Belov, A.: The first harmonic of cosmic ray anisotropy in the convection-diffusion model, Proc. 20th ICRC, 4, 119-122, 1987.

Belov, A. V., Dorman, L. I., Eroshenko, E. A., Oleneva, V. A.: Cosmic ray gradient variations in 1974 , Proc. 20 -th ICRC, 4, 137139, 1987.

Belov, A. V., Dorman, L. I., Eroshenko, E. A., Iucci, N., Villoresi, G., and Yanke, V. G.: Search for Predictors of Forbush Decreases, Proc. of 24-th ICRC, 4, 888-891, 1995.

Belov, A., Bieber, J., Eroshenko, E., Evenson, P., Pyle, R., and Yanke, V.: Pitch-angle features in cosmic rays in advance of se- 
vere magnetic storms: neutron monitor observations, Proc. 27-th ICRC, 9, 3507-3510, 2001a.

Belov, A., Eroshenko, E., Oleneva, V., Struminsky, A., and Yanke, V.: What determines the magnitude of Forbush decreases?, Adv. Space Res., 27, 625-630, 2001b.

Belov, A., Bieber, J., Eroshenko, E., Evenson, P., Pyle, R., and Yanke, V.: Cosmic Ray anisotropy before and during the passage of major solar wind disturbances, Adv. Space Res.., 31, 919-924, 2003.

Belov, A., Baisultanova, L., Eroshenko, E., Mavromichalaki, H., Yanke, V., Pchelkin, V., Plainaki, C., and Mariatos, G.: Magnetospheric effects in cosmic rays during the unique magnetic storm on November 2003, J. Geophys. Res., 110, A09S20, doi:10.1029/2005JA011067, 2005.

Belov, A., Eroshenko, E., Oleneva, V., and Yanke, V.: Connection of Forbush effects to the X-ray flares, J. Atmos. Solar-Terr. Phys., 70, 342-350, 2008.

Chen, J. and Bieber, W.: Cosmic ray anisotropies and gradients in three dimensions, Astrophys. J., 405, 375-389, 1993.

Eroshenko, E., Belov, A., Mavromichalaki, H, Mariatos, G., Oleneva, V., Plainaki, C., and Yanke, V.: Cosmic-Ray Variations During the Two Greatest Bursts of Solar Activity in the 23rd Solar Cycle, Solar Phys., 224, 345-358, 2004.
Keil, W.: Radiation Effects on Spacecraft and Countermeasures, online Proc. 2nd ESA SWW, http://www.esa-spaceweather.net/ spweather/workshops/eswII/proc/Session4/Presentation_KEIL. pps, 2005.

Krymsky, G. F.: Diffusion mechanism of the diurnal cosmic ray variation, Geomagn. Aeronomy, 4, 977-986, 1964.

Leerungnavarat, K. and Ruffolo, D., and Bieber, J. W.: Loss cone precursors to Forbush decreases and advance warning of space weather effects, Astrophys. J., 593, 587-596, 2003.

Mavromichalaki, H., Papaioannou, A., Petrides, A., Assimakopoulos, B., Sarlanis, C., and Souvatzoglou, G.: Cosmic Ray Events Related to Solar Activity at the Athens neutron monitor station for the period 2000-2003, J. Modern Phys. A, 20, 6714-6716, 2005.

Papaioannou, A., Gerontidou, M., Mariatos, G., Mavromichalaki, H., Plainaki, C., Eroshenko, E., Belov, A., and Yanke, V.: Unusually extreme cosmic ray events in July 2005, online Proc. 2n ESA SWW, http://www.esa-spaceweather.net/spweather/workshops/ eswwII/proc/Session1/SESWW-Papaioannou-Poster-pdf, 2005.

Papaioannou, A., Belov, A., Mavromichalaki, H., and Eroshenko, E.: The unusual cosmic ray variations in July 2005 resulted from western and behind the limb solar activity, Adv. Space. Res., 43, 582-588, 2009.

Ruffolo, D., Bieber, J., Evenson, P., and Pyle, R.: Precursors to Forbush decreases and space weather prediction, Proc. 26th ICRC, 6, 440-443, 1999. 